\title{
An Explicit Numerical Method for the Fractional Cable Equation
}

\author{
J. Quintana-Murillo and S. B. Yuste \\ Departamento de Física, Universidad de Extremadura, 06071 Badajoz, Spain \\ Correspondence should be addressed to S. B. Yuste, santos@unex.es
}

Received 27 April 2011; Accepted 30 June 2011

Academic Editor: Fawang Liu

Copyright ( 2011 J. Quintana-Murillo and S. B. Yuste. This is an open access article distributed under the Creative Commons Attribution License, which permits unrestricted use, distribution, and reproduction in any medium, provided the original work is properly cited.

An explicit numerical method to solve a fractional cable equation which involves two temporal Riemann-Liouville derivatives is studied. The numerical difference scheme is obtained by approximating the first-order derivative by a forward difference formula, the Riemann-Liouville derivatives by the Grünwald-Letnikov formula, and the spatial derivative by a three-point centered formula. The accuracy, stability, and convergence of the method are considered. The stability analysis is carried out by means of a kind of von Neumann method adapted to fractional equations. The convergence analysis is accomplished with a similar procedure. The von-Neumann stability analysis predicted very accurately the conditions under which the present explicit method is stable. This was thoroughly checked by means of extensive numerical integrations.

\section{Introduction}

Fractional calculus is a key tool for solving some relevant scientific problems in physics, engineering, biology, chemistry, hydrology, and so on [1-6]. A field of research in which the fractional formalism has been particularly useful is that related to anomalous diffusion processes [1, 7-13]. This kind of process is singularly abundant and important in biological media [14-16]. In this context, the electrodiffusion of ions in neurons is an anomalous diffusion problem to which the fractional calculus has recently been applied. The precise origin of the anomalous character of this diffusion process is not clear (see [17] and references therein), but in any case the consideration of anomalous diffusion in the modeling of electrodiffusion of ions in neurons seems pertinent. This problem has been addressed recently by Langlands et al. $[17,18]$. An equation that plays a key role in their analysis is the following fractional cable (or telegrapher's or Cattaneo) equation (model II):

$$
\frac{\partial u}{\partial t}=\frac{\partial^{1-\gamma_{1}}}{\partial t^{1-\gamma_{1}}}\left(K \frac{\partial^{2} u}{\partial x^{2}}\right)-\mu^{2} \frac{\partial^{1-\gamma_{2}} u}{\partial t^{1-\gamma_{2}}}
$$


where

$$
\frac{\partial^{\gamma}}{\partial t^{\gamma}} f(t) \equiv \frac{1}{\Gamma(n-\gamma)} \frac{d^{n}}{d \tau^{n}} \int_{0}^{t} d \tau \frac{f(\tau)}{(t-\tau)^{1+\gamma-n}}
$$

with $n-1<\gamma<n$ and $n=$ integer, is the Riemann-Liouville fractional derivative. Here $u$ is the difference between the membrane potential and the resting membrane potential, $\gamma_{1}$ is the exponent characterizing the anomalous flux of ions along the nerve cell, and $\gamma_{2}$ is the exponent characterizing the anomalous flux across the membrane $[17,18]$. Some earlier fractional cable equations were discussed in $[19,20]$.

A variety of analytical and numerical methods to solve many classes of fractional equations have been proposed and studied over the last few years [10, 21-30]. Of the numerical methods, finite difference methods have been particularly fruitful [31-38]. These methods can be broadly classified as explicit or implicit [39]. An implicit method for dealing with (1.1) has recently been considered by Liu et al. [38]. Although implicit methods are more cumbersome than explicit methods, they usually remain stable over a larger range of parameters, especially for large timesteps, which makes them particularly suitable for fractional diffusion problems. Nevertheless, explicit methods have some features that make them widely appreciated [32,39]: flexibility, simplicity, small computational demand, and easy generalization to spatial dimensions higher than one. Unfortunately, they can become unstable in some cases, so that it is of great importance to determine the conditions under which these methods are stable. In this paper we will discuss an explicit finite difference scheme for solving the fractional cable equation, which is close to the methods studied in $[32,33]$. We shall address two main questions: (i) whether this kind of method can cope with fractional equations involving different fractional derivatives, such as the fractional cable equation; (ii) whether the von Neumann stability analysis put forward in $[32,34]$ is suitable for this kind of equation.

\section{The Numerical Method}

Henceforth, we will use the notation $x_{j}=j \Delta x, t_{m}=m \Delta t$, and $u\left(x_{j}, t_{m}\right)=u_{j}^{(m)} \simeq U_{j}^{(m)}$, where $U_{j}^{(m)}$ is the numerical estimate of the exact solution $u(x, t)$ at $x=x_{j}$ and $t=t_{m}$.

In order to get the numerical difference algorithm, we discretize the continuous differential and integro-differential operators as follows. For the discretization of the fractional Riemann-Liouville derivative we use the Grünwald-Letnikov formula

$$
\frac{d^{1-\gamma}}{d t^{1-\gamma}} u(x, t)=\Delta_{t}^{1-\gamma} u\left(x, t_{m}\right)+O(\Delta t)
$$

with

$$
\begin{aligned}
\Delta_{t}^{\alpha} f\left(t_{m}\right) & =\frac{1}{(\Delta t)^{\alpha}} \sum_{k=0}^{m} \omega_{k}^{(\alpha)} f\left(t_{m-k}\right), \\
\omega_{k}^{(\alpha)} & =\left(1-\frac{1+\alpha}{k}\right) \omega_{k-1}^{(\alpha)},
\end{aligned}
$$


and $\omega_{0}^{(\alpha)}=1$. These coefficients come from the generating function [40]

$$
(1-z)^{\alpha}=\sum_{k=0}^{\infty} \omega_{k}^{(\alpha)} z^{k}
$$

To discretize the integer derivatives we use standard formulas: for the second-order spatial derivative we employ the three-point centered formula

$$
\frac{\partial^{2}}{\partial x^{2}} u\left(x_{j}, t_{m}\right)=\Delta_{x}^{2} u_{j}^{(m)}+O(\Delta x)^{2}
$$

with

$$
\Delta_{x}^{2} u_{j}^{(m)}=\frac{u\left(x_{j+1}, t_{m}\right)-2 u\left(x_{j}, t_{m}\right)+u\left(x_{j-1}, t_{m}\right)}{(\Delta x)^{2}}
$$

and for the first-order time derivative we use the forward derivative

$$
\frac{\partial}{\partial t} u\left(x_{j}, t_{m}\right)=\delta_{t} u_{j}^{m+1}+O(\Delta t)
$$

where

$$
\delta_{t} u_{j}^{m+1}=\frac{u\left(x_{j}, t_{m+1}\right)-u\left(x_{j}, t_{m}\right)}{\Delta t} .
$$

Inserting (2.1), (2.5), and (2.7) into (1.1), one gets

$$
\delta_{t} u_{j}^{(m+1)}-K \Delta_{t}^{1-\gamma_{1}}\left(\Delta_{x}^{2} u_{j}^{(m)}\right)+\mu^{2} \Delta_{t}^{1-\gamma_{2}} u_{j}^{(m)}=T(x, t),
$$

where, as can easily be proved, the truncating error $T(x, t)$ is

$$
T(x, t)=O(\Delta t)+O(\Delta x)^{2} .
$$

Neglecting the truncating error we get the finite difference scheme we are seeking:

$$
\delta_{t} U_{j}^{(m+1)}-K \Delta_{t}^{1-\gamma_{1}} \Delta_{x}^{2} U_{j}^{(m)}+\mu^{2} \Delta_{t}^{1-\gamma_{2}} U_{j}^{(m)}=0
$$

that is,

$$
U_{j}^{(m+1)}=U_{j}^{(m)}+S \sum_{k=0}^{m} \omega_{k}^{1-\gamma_{1}}\left(U_{j+1}^{(m-k)}-2 U_{j}^{(m-k)}+U_{j-1}^{(m-k)}\right)-\mu^{2}(\Delta t)^{\gamma_{2}} \sum_{k=0}^{m} \omega_{k}^{1-\gamma_{2}} U_{j}^{(m-k)},
$$


where

$$
S=K \frac{(\Delta t)^{\gamma_{1}}}{(\Delta x)^{2}}
$$

To test this algorithm, we solved (1.1) in the interval $-L / 2 \leq x \leq L / 2$, with absorbing boundary conditions, $u(x=-L / 2, t)=u(x=L / 2, t)=0$, and initial condition given by a Dirac's delta function centered at $x=0: u(x, 0)=\delta(x)$. The exact solution of this problem for $L \rightarrow \infty$ is [17]

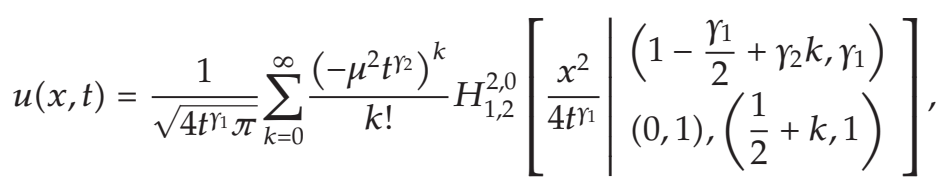

where $H$ denotes the Fox $H$ function $[10,41]$. In our numerical procedure, the exact initial condition $u(x, 0)=\delta(x)$ is approximated by

$$
u\left(x_{j}, 0\right)= \begin{cases}\frac{1}{\Delta x}, & j=0 \\ 0, & j \neq 0\end{cases}
$$

The explicit difference scheme (2.12) is tested by comparing the analytical solution with the numerical solution for several cases of the problem described following (2.13) with different values of $\gamma_{1}$ and $\gamma_{2}$. We have computed the analytical solution by means of (2.14) truncating the series at $k=20$. The corresponding Fox $H$ function was evaluated by means of the series expansion described in $[10,41]$ truncating the infinite series after the first 50 terms. In Figures 1 and 2 we show the analytical and numerical solution for two values of $\gamma_{1}$ and $\gamma_{2}$ at $x=0$ and $x=0.5$. The differences between the exact and the numerical solution are shown in Figures 3 and 4 . One sees that, except for very short times, the agreement is quite good. The large value of the error for small times is due in part to the approximation of the Dirac's delta function at $x=0$ by (2.15). This is clearly appreciated when noticing the quite different scales of Figures 3 and 4: the error is much smaller for $x=0.5$ than for $x=0$. For the cases with $\gamma_{1}=1 / 2$ we used a smaller value of $\Delta t$ and, simultaneously, a larger value of $\Delta x$ than for the cases with $\gamma_{1}=1$ in order to keep the numerical scheme stable. This issue will be discussed in Section 3 .

\section{Stability}

As usual for explicit methods, the present explicit difference scheme (2.12) is not unconditionally stable, that is, for any given set of values of $\gamma_{1}, \gamma_{2}, \mu$, and $K$ there are choices of $\Delta x$ and $\Delta t$ for which the method is unstable. Therefore, it is important to determine the conditions under which the method is stable. To this end, here we shall employ the fractional von Neumann stability analysis (or Fourier analysis) put forward in [32] (see also [33-35]). The question we address is to what extent this procedure is valid for fractional diffusion equations that involve fractional derivatives of different order.

Proceeding as [32], we start by recognizing that the solution of our problem can be written as the linear combination of subdiffusive modes, $u_{j}^{(m)}=\sum_{q} \zeta_{q}^{(m)} e^{i q j \Delta x}$, where the 


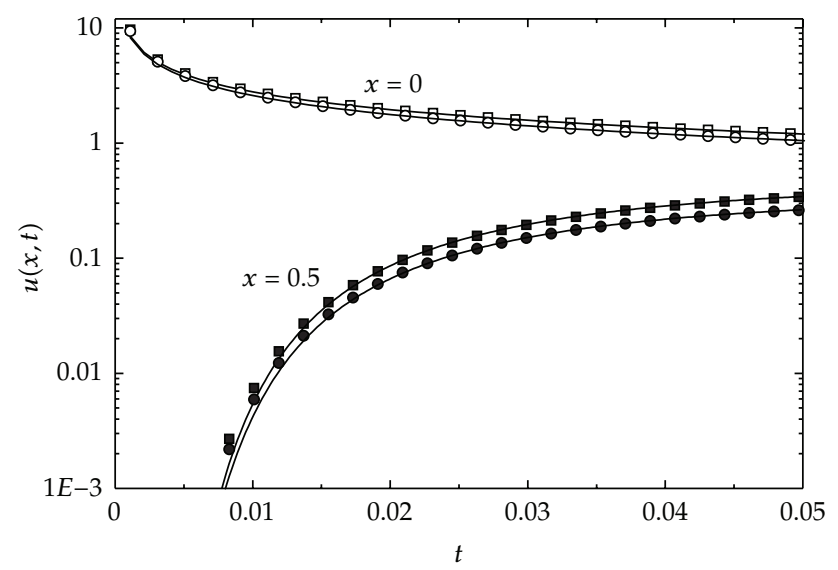

Figure 1: Numerical solution at the mid-point $x=0$ (hollow symbols) and $x=0.5$ (filled symbols) of the fractional cable equation for $\gamma_{1}=1$ and $\gamma_{2}=1$ (squares) and $\gamma_{2}=1 / 2$ (circles) with $\Delta x=1 / 20, \Delta t=10^{-4}$, $K=\mu=1$, and $L=5$. Lines are the exact solutions given by (2.14).

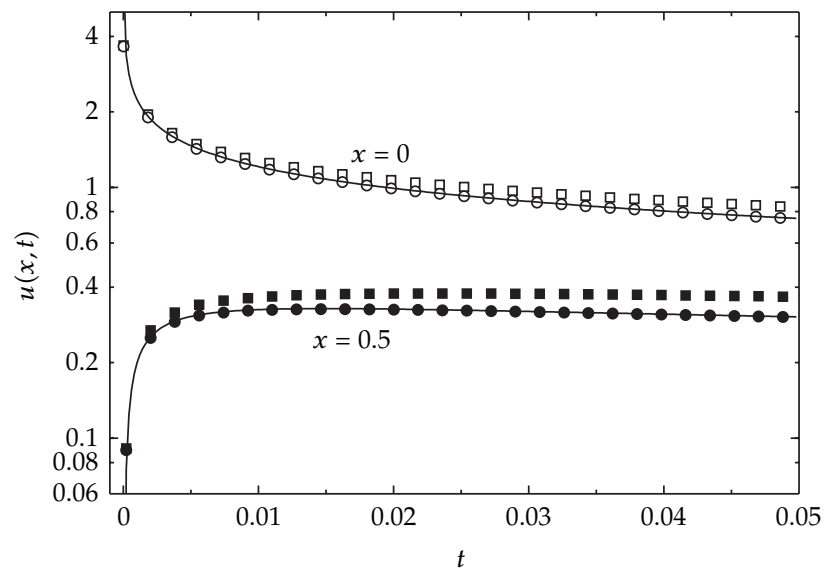

Figure 2: Numerical solution at the mid-point $x=0$ (hollow symbols) and $x=0.5$ (filled symbols) of the fractional cable equation for $\gamma_{1}=1 / 2$ and $\gamma_{2}=1$ (squares) and $\gamma_{2}=1 / 2$ (circles) with $\Delta x=1 / 10, \Delta t=10^{-5}$, $K=\mu=1$, and $L=5$. Lines are the exact solutions given by (2.14).

sum is over all the wave numbers $q$ supported by the lattice. Therefore, following the von Neumann ideas, we reduce the problem of analyzing the stability of the solution to the problem of analyzing the stability of a single generic subdiffusion mode, $\zeta^{(m)} e^{i q j \Delta x}$. Inserting this expression into (2.12) one gets

$$
\zeta^{(m+1)}=\zeta^{(m)}+S \sum_{k=0}^{m} \omega_{k}^{\left(1-\gamma_{1}\right)}\left(e^{i q \Delta x}-2+e^{-i q \Delta x}\right) \zeta^{(m-k)}-\mu^{2}(\Delta t)^{\gamma_{2}} \sum_{k=0}^{m} \omega_{k}^{\left(1-\gamma_{2}\right)} \zeta^{(m-k)} .
$$

The stability of the mode is determined by the behavior of $\zeta^{(m)}$. Writing

$$
\zeta^{(m+1)}=\xi \zeta^{(m)}
$$




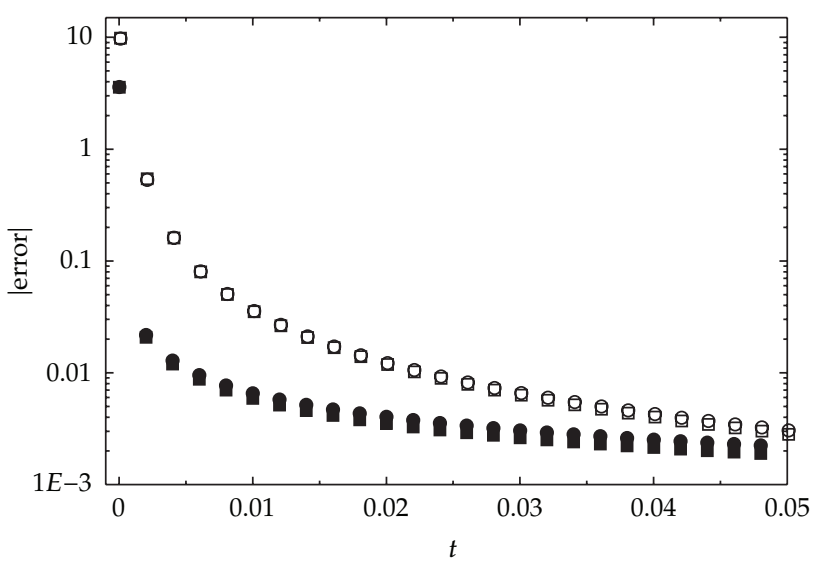

Figure 3: Absolute error $\left|U_{j}^{(m)}-u_{j}^{(m)}\right|$ of the numerical method for the problems considered in Figures 1 and 2 at $x=0$. Squares: $\gamma_{2}=1$; circles: $\gamma_{2}=1 / 2$; hollow symbols: $\gamma_{1}=1$; filled symbols: $\gamma_{1}=1 / 2$.

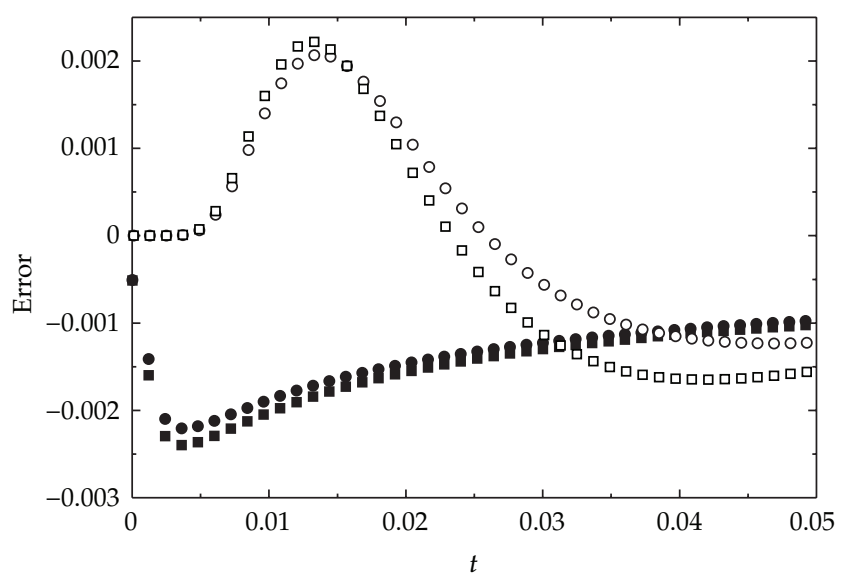

Figure 4: Error $U_{j}^{(m)}-u_{j}^{(m)}$ of the numerical method for the problem considered in Figures 1 and 2 at $x=0.5$. Squares: $\gamma_{2}=1$; circles: $\gamma_{2}=1 / 2$; hollow symbols: $\gamma_{1}=1$; filled symbols: $\gamma_{1}=1 / 2$.

and assuming that the amplification factor $\xi$ of the subdiffusive mode is independent of time, we get

$$
\xi=1+S \sum_{k=0}^{m} \omega_{k}^{\left(1-\gamma_{1}\right)}\left(e^{i q \Delta x}-2+e^{-i q \Delta x}\right) \xi^{-k}-\mu^{2}(\Delta t)^{\gamma_{2}} \sum_{k=0}^{m} \omega_{k}^{\left(1-\gamma_{2}\right)} \xi^{-k}
$$

If $|\xi|>1$ for some $q$, the temporal factor of the solution grows to infinity [c.f., (3.2)], and the mode is unstable. Considering the extreme value $\xi=-1$, one gets from (3.3) that the numerical method is stable if this inequality holds:

$$
\bar{S} \leq S_{\times}^{m}=\frac{-2+\mu^{2}(\Delta t)^{\gamma_{2}} \sum_{k=0}^{m} \omega_{k}^{\left(1-\gamma_{2}\right)}(-1)^{k}}{-4 \sum_{k=0}^{m} \omega_{k}^{\left(1-\gamma_{1}\right)}(-1)^{k}}
$$


where

$$
\bar{S}=S \sin ^{2}\left(\frac{q \Delta x}{2}\right)
$$

If one defines $S_{\times}=\lim _{m \rightarrow \infty} S_{\times}^{m}$, one gets

$$
\bar{S} \leq S_{\times}=\frac{-2+\mu^{2}(\Delta t)^{\gamma_{2}} \sum_{k=0}^{\infty} \omega_{k}^{\left(1-\gamma_{2}\right)}(-1)^{k}}{-4 \sum_{k=0}^{\infty} \omega_{k}^{1-\gamma_{1}}(-1)^{k}} .
$$

But from (2.4) with $z=-1$ one sees that $\sum_{k=1}^{\infty}(-1)^{k} \omega_{k}^{(1-\gamma)}=2^{1-\gamma}$, so that

$$
S_{\times}=\frac{2^{\gamma_{2}}-\mu^{2}(\Delta t)^{\gamma_{2}}}{2^{2+\gamma_{2}-\gamma_{1}}}
$$

Therefore, because $S \leq \bar{S}$, we find that a sufficient condition for the present method to be stable is that $S \leq S_{\times}$. In Figures 5 and 6 we show two representative examples of the problem considered in Figure 2 but for two values of $S$, respectively, larger and smaller than the stability bound provided by (3.7). One sees that the value of $S$ that one chooses is crucial: when $S$ is smaller than $S_{\times}$one is inside the stable region and gets a sensible numerical solution (Figure 5); otherwise one gets an evidently unstable and nonsensical solution (Figure 6).

\section{Numerical Check of the Stability Analysis}

In this section we describe a comprehensive check of the validity of our stability analysis by using many different values of the parameters $\gamma_{1}, \gamma_{2}, \Delta t$, and $\Delta x$ and testing whether the stability of the numerical method is as predicted by (3.7). Without loss of generality, we assume $\mu=K=1$ in all cases. We proceed in the following way. First, we choose a set of values of $\gamma_{1}, \gamma_{2}, \Delta x$, and $S$ and integrate the corresponding fractional cable equation. If

$$
\left|U_{j}^{m-1}-U_{j}^{m}\right|>\lambda
$$

for $\lambda=10$ within the first 1000 integrations, then we say the method is unstable; otherwise, we label the method as stable. We generated Figure 7 by starting the integration for values of $S$ well below the theoretical stability limit given by (3.7) and kept increasing its value by 0.001 until condition (4.1) was first reached. The last value for which the method was stable is recorded and plotted in Figure 7. The limit value $\lambda=10$ is arbitrary, but choosing any other reasonable value does not significantly change these plots.

\section{Convergence Analysis}

In this section we show that the present numerical method is convergent, that is, that the numerical solution converges towards the exact solution when the size of the spatiotemporal 


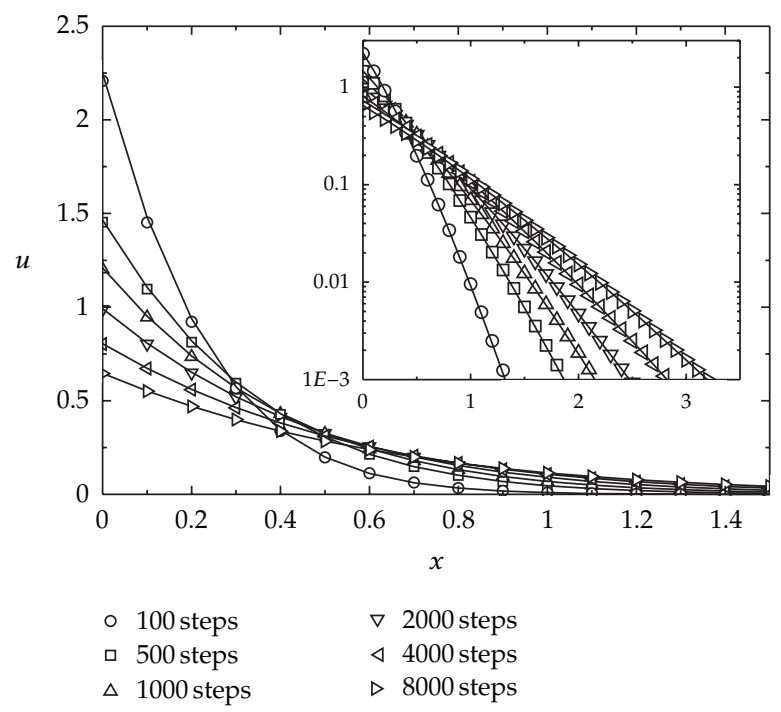

Figure 5: Exact solution (lines) and numerical solution (symbols) provided by our method for the fractional cable equation with $\gamma_{1}=0.5$ and $\gamma_{2}=0.5$ for different numbers of timesteps when $\Delta x=1 / 10$, $\Delta t=10^{-5}, K=\mu=1, L=5$, and $S=(\Delta t)^{\gamma_{1}} /(\Delta x)^{2}=0.316$. This case is inside the stability region because $S$ is smaller than the stability limit $S_{\times}=\left(2^{\gamma_{2}}-\mu^{2}(\Delta t)^{\gamma_{2}}\right) /\left(2^{2+\gamma_{2}-\gamma_{1}}\right) \simeq 0.352 \ldots$ provided by (3.7). The inset shows the results on logarithmic scale.

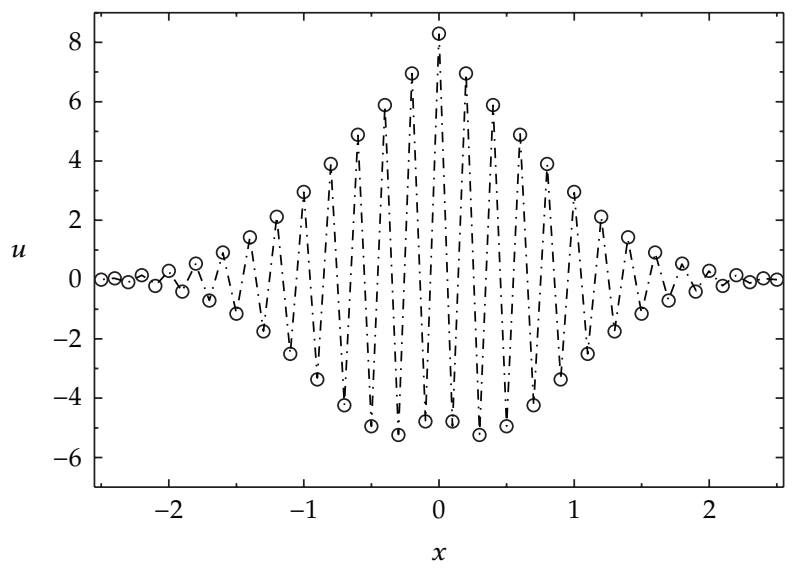

Figure 6: Numerical solution (circles) provided by our explicit method for the fractional cable equation with $\gamma_{1}=0.5$ and $\gamma_{2}=0.5$ after 100 timesteps when $\Delta x=1 / 10, \Delta t=1.3 \times 10^{-5}, K=\mu=1, L=5$, and $S=(\Delta t)^{\gamma} /(\Delta x)^{2}=0.36$. Note that this value is larger than the stability limit $S_{\times}=\left(2^{\gamma_{2}}-\mu^{2}(\Delta t)^{\gamma_{2}}\right) /\left(2^{2+\gamma_{2}-\gamma_{1}}\right) \simeq$ $0.352 \ldots$ provided by (3.7). The broken line is to guide the eye.

discretization goes to zero. Let us define $e_{j}^{(k)}$ as the difference between the exact and numerical solutions at the point $\left(x_{j}, t_{m}\right): e_{j}^{(k)}=u_{j}^{(k)}-U_{j}^{(k)}$. Taking into account (2.9) and (2.11), one gets the equation that describes how this difference evolves:

$$
\begin{aligned}
e_{j}^{(m+1)} & -e_{j}^{(m)}-S \sum_{k=0}^{m} \omega_{k}^{\left(1-\gamma_{1}\right)}\left(e_{j+1}^{(m-k)}-2 e_{j}^{(m-k)}+e_{j-1}^{(m-k)}\right)+\mu^{2}(\Delta t)^{\gamma_{2}} \sum_{k=0}^{m} \omega_{k}^{\left(1-\gamma_{2}\right)} e_{j}^{(m-k)} \\
& =T\left(x_{j}, t_{k}\right) \equiv T_{j}^{(m)} .
\end{aligned}
$$




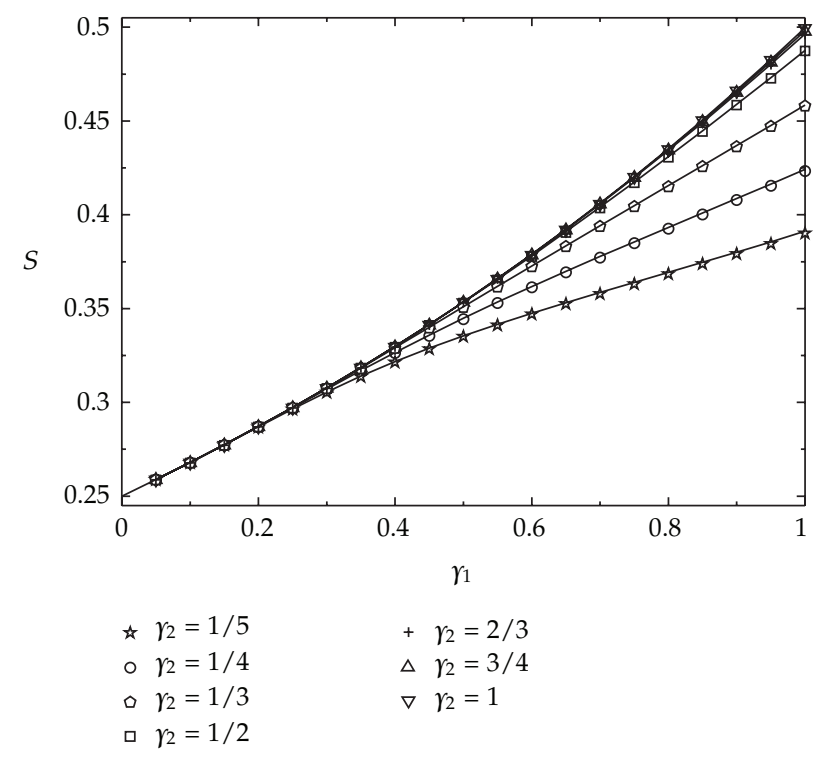

Figure 7: Stability bound $S$ versus $\gamma_{1}$ for several values of $\gamma_{2}$ where $\Delta x=1 / 20$, and $K=\mu=1$. Symbols are numerical estimates. Lines correspond to the theoretical prediction (3.7).

As we did in the previous section for $U_{j}^{(k)}$, we write $e_{j}^{(k)}$ and $T_{j}^{(m)}$ as a combination of (sub) diffusion modes, $e_{j}^{(k)}=\sum_{q} \zeta_{q}^{(k)} e^{i q j \Delta x}$ and $T_{j}^{(m)}=\sum_{q} x_{q}^{(m)} e^{i q j \Delta x}$, and analyze the convergence of a single but generic $q$-mode $[36,39,42]$. Therefore, replacing $e_{j}^{(k)}$ by $\zeta^{(k)} e^{i q j \Delta x}$ and $T_{j}^{(m)}$ by $x^{(m)} e^{i q j \Delta x}$ in (5.1), we get

$$
\zeta^{(m+1)}=\zeta^{(m)}+\bar{S} \sum_{k=0}^{m} \omega_{k}^{\left(1-\gamma_{1}\right)} \zeta^{(m-k)}+\mu^{2}(\Delta t)^{\gamma_{2}} \sum_{k=0}^{m} \omega_{k}^{\left(1-\gamma_{2}\right)} \zeta^{(m-k)}+x^{(m)} .
$$

Now we will prove by induction that $\left|\zeta^{(m)}\right|=O(\Delta t)+O(\Delta x)^{2}$ for all $m$. To start, $U_{j}^{(0)}$ satisfies the initial condition by construction, so that $e_{j}^{(0)}=0$. This means that $\zeta^{(0)}=0$. Therefore, from (5.2) one gets $\zeta^{(1)}=\chi^{(0)}$. But from (2.10) one knows that $\left|T_{j}^{(0)}\right|=\left|\chi^{(0)}\right|=$ $O(\Delta t)+O(\Delta x)^{2}$, so that $\left|\zeta^{(1)}\right|=O(\Delta t)+O(\Delta x)^{2}$. Let us now assume that $\left|\zeta^{(k)}\right|=O(\Delta t)+$ $O(\Delta x)^{2}$ holds for $k=1, \ldots, m$. Then we will prove that $\left|\zeta^{(m+1)}\right|=O(\Delta t)+O(\Delta x)^{2}$. From (5.2) we obtain

$$
\left|\zeta^{(m+1)}\right| \leq\left|x^{(m)}\right|+\left|\zeta^{(m)}\right|+\bar{S}\left|\zeta^{\{m\}}\right| \sum_{k=0}^{m}\left|\omega_{k}^{\left(1-\gamma_{1}\right)}\right|-\mu^{2}(\Delta t)^{\gamma_{2}}\left|\zeta^{\{m\}}\right| \sum_{k=0}^{m}\left|\omega_{k}^{\left(1-\gamma_{2}\right)}\right|,
$$

where $\left|\zeta^{\{m\}}\right|$ is the maximum value of $\left|\zeta^{(k)}\right|$ for $k=0, \ldots, m$. Taking into account (2.4), using the value $z=1$, and because $\omega_{0}^{(\alpha)}=1$, it is easy to see that $\sum_{k=1}^{\infty} \omega_{k}^{(\alpha)}=-1$ or, equivalently, $\sum_{k=1}^{\infty}\left|\omega_{k}^{(\alpha)}\right|=1$ since $\omega_{k}^{(\alpha)}<0$ for $k \geq 1$ (see (2.3)). Therefore $\sum_{k=0}^{m}\left|\omega_{k}^{(1-\gamma)}\right|$ is bounded (in 
fact, it is smaller than 2). Using this result in (5.3), together with $\left|\zeta^{(k)}\right| \leq C\left(\Delta t+(\Delta x)^{2}\right)$ and $\left|\chi^{(k)}\right| \leq C\left(\Delta t+(\Delta x)^{2}\right)$, we find that

$$
\left|\zeta^{(m+1)}\right| \leq C\left(\Delta t+(\Delta x)^{2}\right)
$$

Therefore the amplitude of the subdiffusive modes goes to zero when the spatiotemporal mesh goes to zero. Employing the Parseval relation, this means that the norm of the error $\left\|e^{(k)}\right\|^{2} \equiv \sum_{j}\left|e_{j}^{(k)}\right|^{2}=\sum_{q}\left|\zeta_{q}^{(k)}\right|^{2}$ goes to zero when $\Delta t$ and $\Delta x$ go to zero. This is what we aimed to prove.

\section{Conclusions}

An explicit method for solving a kind of fractional diffusion equation that involves several fractional Riemann-Liouville derivatives, which are approximated by means of the Grünwald-Letnikov formula, has been considered. The method was used to solve a class of equations of this type (fractional cable equations) with free boundary conditions, Dirac's delta initial condition, and different fractional exponents. The error of the numerical method is compatible with the truncating error, which is of order $O(\Delta t)+O(\Delta x)^{2}$. It was also proved that the method is convergent. Besides, it was also found that a fractional von-Neumann stability analysis, which provides very precise stability conditions for standard fractional diffusion equations, leads also to a very accurate estimate of the stability conditions for cable equations.

\section{References}

[1] R. Klages, G. Radons, and I. M. Sokolov, Eds., Anomalous Transport: Foundations and Applications, Elsevier, Amsterdam, The Netherlands, 2008.

[2] I. Podlubny, Fractional Differential Equations, vol. 198, Academic Press, San Diego, Calif, USA, 1999.

[3] R. Hilfer, Ed., Applications of Fractional Calculus in Physics, World Scientific, Singapore, 2000.

[4] A. A. Kilbas, H. M. Srivastava, and J. J. Trujillo, Theory and Applications of Fractional Differential Equations, vol. 204, Elsevier, Amsterdam, The Netherlands, 2006.

[5] R. L. Magin, O. Abdullah, D. Baleanu, and X. J. Zhou, "Anomalous diffusion expressed through fractional order differential operators in the Bloch-Torrey equation," Journal of Magnetic Resonance, vol. 190, no. 2, pp. 255-270, 2008.

[6] D. Baleanu, "Fractional variational principles in action," Physica Scripta, vol. 2009, no. T136, Article ID 014006, 4 pages, 2009.

[7] S. B. Yuste and L. Acedo, "Some exact results for the trapping of subdiffusive particles in one dimension," Physica A, vol. 336, no. 3-4, pp. 334-346, 2004.

[8] S. B. Yuste and K. Lindenberg, "Trapping reactions with subdiffusive traps and particles characterized by different anomalous diffusion exponents," Physical Review E, vol. 72, no. 6, Article ID 061103, 8 pages, 2005.

[9] S. B. Yuste and K. Lindenberg, "Subdiffusive target problem: survival probability," Physical Review E, vol. 76, no. 5, Article ID 051114, 6 pages, 2007.

[10] R. Metzler and J. Klafter, "The random walk's guide to anomalous diffusion: a fractional dynamics approach," Physics Reports, vol. 339, no. 1, p. 77, 2000.

[11] R. Metzler and J. Klafter, "The restaurant at the end of the random walk: recent developments in the description of anomalous transport by fractional dynamics," Journal of Physics A, vol. 37, no. 31, pp. R161-R208, 2004. 
[12] R. Metzler, E. Barkai, and J. Klafter, "Anomalous diffusion and relaxation close to thermal equilibrium: A fractional Fokker-Planck equation approach," Physical Review Letters, vol. 82, no. 18, pp. 3563-3567, 1999.

[13] S. B. Yuste, E. Abad, and K. Lindenberg, "A reaction-subdiffusion model of morphogen gradient formation," Physical Review E, vol. 82, no. 6, Article ID 061123, 9 pages, 2010.

[14] J. A. Dix and A. S. Verkman, "Crowding effects on diffusion in solutions and cells," Annual Review of Biophysics, vol. 37, pp. 247-263, 2008.

[15] I. Y. Wong, M. L. Gardel, D. R. Reichman et al., "Anomalous Diffusion Probes Microstructure Dynamics of Entangled F-Actin Networks," Physical Review Letters, vol. 92, no. 17, Article ID 178101, 4 pages, 2004.

[16] J.-H. Jeon, V. Tejedor, S. Burov et al., “In Vivo Anomalous Diffusion and Weak Ergodicity Breaking of Lipid Granules," Physical Review Letters, vol. 106, no. 4, Article ID 048103, 4 pages, 2011.

[17] T. A. M. Langlands, B. I. Henry, and S. L. Wearne, "Fractional cable equation models for anomalous electrodiffusion in nerve cells: infinite domain solutions," Journal of Mathematical Biology, vol. 59, no. 6, pp. 761-808, 2009.

[18] B. I. Henry, T. A. M. Langlands, and S. L. Wearne, "Fractional cable models for spiny neuronal dendrites," Physical Review Letters, vol. 100, no. 12, Article ID 128103, p. 4, 2008.

[19] A. Compte and R. Metzler, "The generalized Cattaneo equation for the description of anomalous transport processes," Journal of Physics A, vol. 30, no. 21, pp. 7277-7289, 1997.

[20] R. Metzler and T. F. Nonnenmacher, "Fractional diffusion, waiting-time distributions, and Cattaneotype equations," Physical Review E, vol. 57, no. 6, pp. 6409-6414, 1998.

[21] S. S. Ray, "Exact solutions for time-fractional diffusion-wave equations by decomposition method," Physica Scripta, vol. 75, no. 1, pp. 53-61, 2007.

[22] S. Momani, A. Odibat, and V. S. Erturk, "Generalized differential transform method for solving a space- and time-fractional diffusion-wave equation," Physics Letters. A, vol. 370, no. 5-6, pp. 379-387, 2007.

[23] H. Jafari and S. Momani, "Solving fractional diffusion and wave equations by modified homotopy perturbation method," Physics Letters. A, vol. 370, no. 5-6, pp. 388-396, 2007.

[24] A. M. A. El-Sayed and M. Gaber, "The Adomian decomposition method for solving partial differential equations of fractal order in finite domains," Physics Letters A, vol. 359, no. 3, pp. 175-182, 2006.

[25] I. Podlubny, A. V. Chechkin, T. Skovranek, Y. Chen, and B. M. Vinagre Jara, "Matrix approach to discrete fractional calculus. II. Partial fractional differential equations," Journal of Computational Physics, vol. 228, no. 8, pp. 3137-3153, 2009.

[26] E. Barkai, "Fractional Fokker-Planck equation, solution, and application," Physical Review E, vol. 63, no. 4, Article ID 046118, 17 pages, 2001.

[27] M. Enelund and G. A. Lesieutre, "Time domain modeling of damping using anelastic displacement fields and fractional calculus," International Journal of Solids and Structures, vol. 36, no. 29, pp. 44474472, 1999.

[28] G. J. Fix and J. P. Roop, “Least squares finite-element solution of a fractional order two-point boundary value problem," Computers \& Mathematics with Applications. An International Journal, vol. 48, no. 7-8, pp. 1017-1033, 2004.

[29] Y. Zheng, C. Li, and Z. Zhao, "A note on the finite element method for the space-fractional advection diffusion equation," Computers \& Mathematics with Applications, vol. 59, no. 5, pp. 1718-1726, 2010.

[30] Y. Zheng, C. Li, and Z. Zhao, "A fully discrete discontinuous Galerkin method for nonlinear fractional Fokker-Planck equation," Mathematical Problems in Engineering, vol. 2010, Article ID 279038, p. 26, 2010.

[31] R. Gorenflo, F. Mainardi, D. Moretti, and P. Paradisi, "Time fractional diffusion: a discrete random walk approach," Nonlinear Dynamics, vol. 29, no. 1-4, pp. 129-143, 2002.

[32] S. B. Yuste and L. Acedo, "An explicit finite difference method and a new von Neumann-type stability analysis for fractional diffusion equations," SIAM Journal on Numerical Analysis, vol. 42, no. 5, pp. 1862-1874, 2005.

[33] S. B. Yuste and J. Quintana-Murillo, "On three explicit difference schemes for fractional diffusion and diffusion-wave equations," Physica Scripta, vol. 2009, no. T136, Article ID 014025, 6 pages, 2009.

[34] S. B. Yuste, "Weighted average finite difference methods for fractional diffusion equations," Journal of Computational Physics, vol. 216, no. 1, pp. 264-274, 2006.

[35] J. Quintana-Murillo and S. B. Yuste, "An explicit difference method for solving fractional diffusion and diffusion-wave equations in the Caputo form," Journal of Computational and Nonlinear Dynamics, vol. 6, no. 2, Article ID 021014, 6 pages, 2011. 
[36] C. M. Chen, F. Liu, I. Turner, and V. Anh, "A Fourier method for the fractional diffusion equation describing sub-diffusion," Journal of Computational Physics, vol. 227, no. 2, pp. 886-897, 2007.

[37] P. Zhuang, F. Liu, V. Anh, and I. Turner, "New solution and analytical techniques of the implicit numerical method for the anomalous subdiffusion equation," SIAM Journal on Numerical Analysis, vol. 46, no. 2, pp. 1079-1095, 2008.

[38] F. Liu, Q. Yang, and I. Turner, "Stability and convergence of two new implicit numerical methods for the fractional cable equation," Journal of Computational and Nonlinear Dynamics, vol. 6, no. 1, Article ID 01109, 7 pages, 2011.

[39] K. W. Morton and D. F. Mayers, Numerical Solution of Partial Differential Equations, Cambridge University Press, Cambridge, UK, 1994.

[40] Ch. Lubich, "Discretized fractional calculus," SIAM Journal on Mathematical Analysis, vol. 17, no. 3, pp. 704-719, 1986.

[41] A. M. Mathai and R. K. Saxena, The H-Function with Applications in Statistics and Other Disciplines, John Wiley \& Sons, New York, NY, USA, 1978.

[42] M. Cui, "Compact finite difference method for the fractional diffusion equation," Journal of Computational Physics, vol. 228, no. 20, pp. 7792-7804, 2009. 


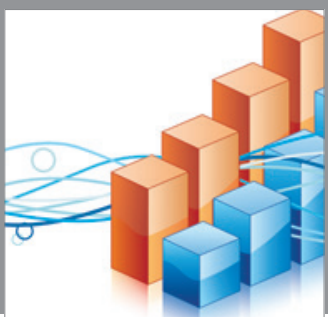

Advances in

Operations Research

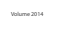

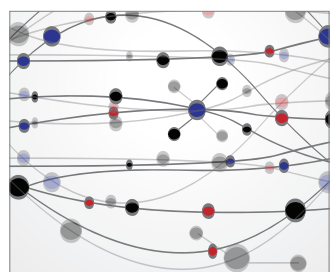

\section{The Scientific} World Journal
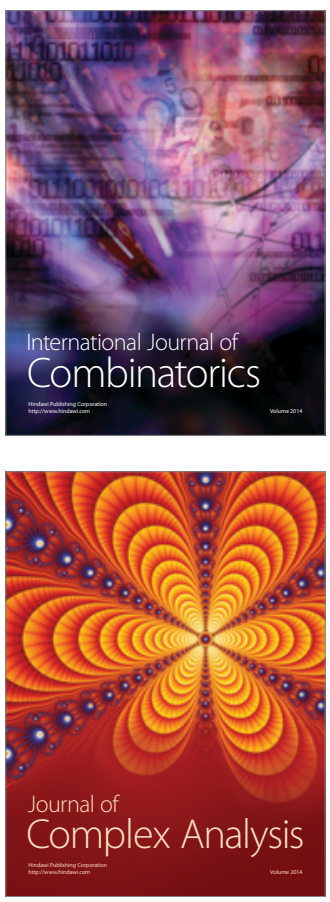

International Journal of

Mathematics and

Mathematical

Sciences
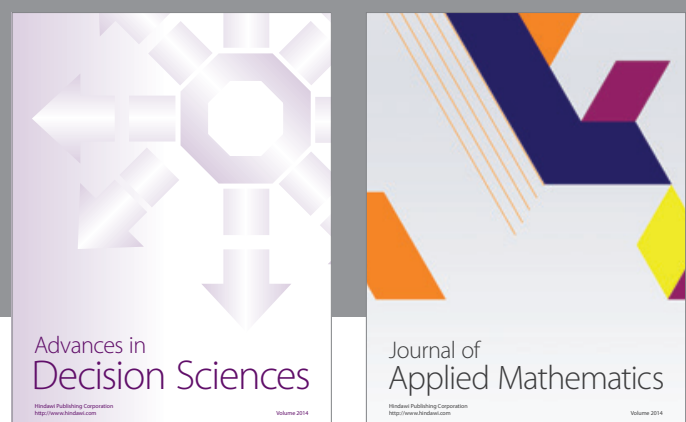

Journal of

Applied Mathematics
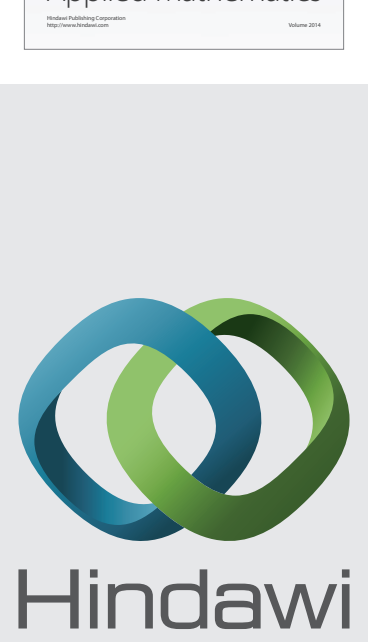

Submit your manuscripts at http://www.hindawi.com
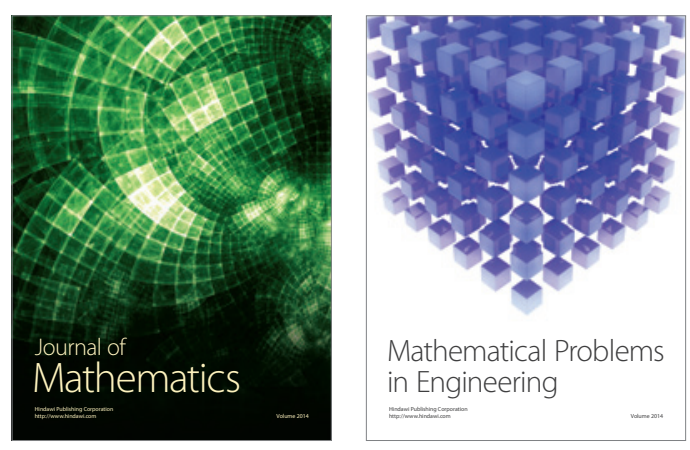

Mathematical Problems in Engineering
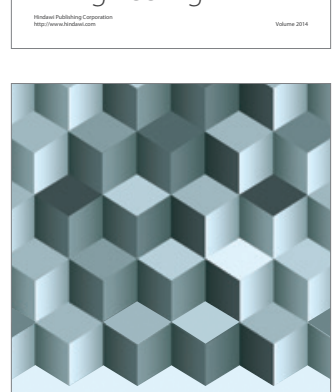

Journal of

Function Spaces
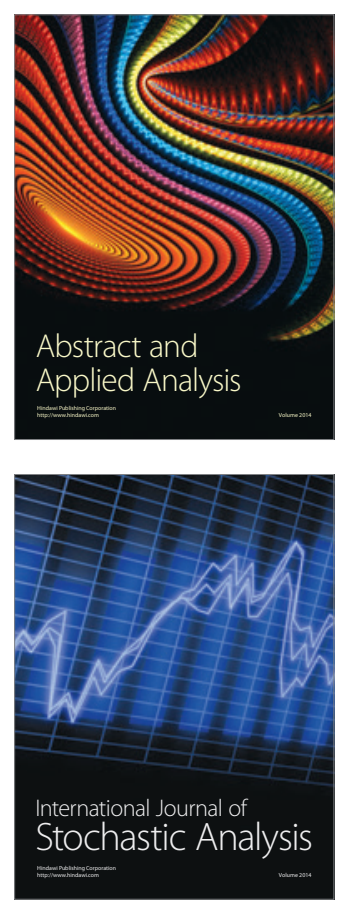

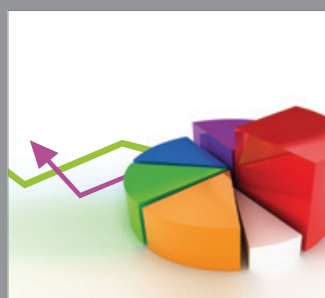

ournal of

Probability and Statistics

Promensencen
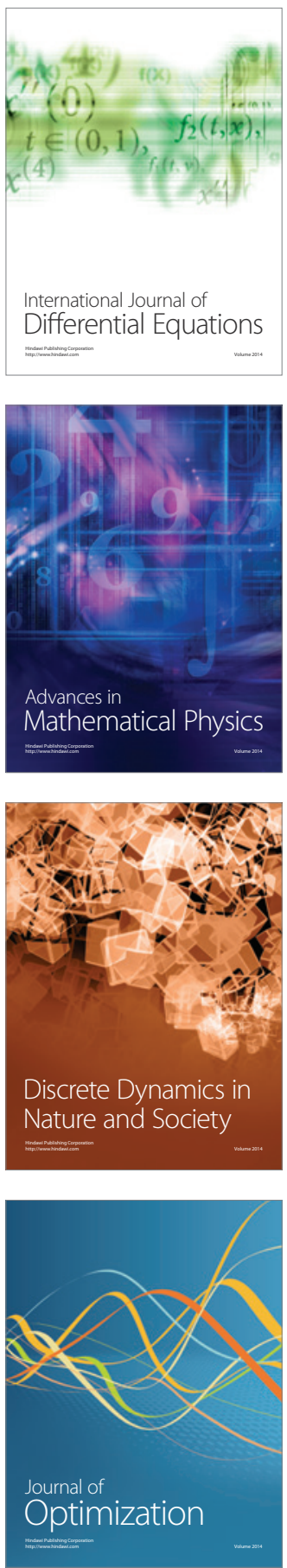